\title{
The 1590-1520 Ma Cachoeirinha magmatic arc and its tectonic implications for the Mesoproterozoic SW Amazonian craton crustal evolution
}

\author{
AMARILDO S. RUIZ ${ }^{1}$, MAURO C. GERALDES ${ }^{2}$, JOÃO B. MATOS ${ }^{1}$, WILSON TEIXEIRA ${ }^{3}$ \\ WILLIAM R. VAN SCHMUS ${ }^{4}$ and RENATA S. SCHMITT ${ }^{2}$ \\ ${ }^{1}$ Departamento de Geologia, Universidade Federal de Mato Grosso, Av. Fernando Correia s/n \\ 09923-900 Cuiabá, MT, Brasil \\ ${ }^{2}$ Tektos - Grupo de Pesquisa em Tectônica, Faculdade de Geologia \\ Universidade do Estado do Rio de Janeiro, Rua São Francisco Xavier 524, Maracanã \\ 20550-013 Rio de Janeiro, RJ, Brasil \\ ${ }^{3}$ Instituto de Geociências, Universidade de São Paulo, Rua do Lago, 562, Cidade Universitária \\ 05508-900 São Paulo, SP, Brasil \\ ${ }^{4}$ Department of Geology, University of Kansas, Lawrence, Kansas 66045 USA \\ Manuscript received on October 10, 2003; accepted for publication on July 21, 2004; \\ contributed by Wilson TeIXeIRA* and William R. VAn Schmus*
}

\begin{abstract}
Isotopic and chemical data of rocks from the Cachoeirinha suite provide new insights on the Proterozoic evolution of the Rio Negro/Juruena Province in SW Amazonian craton. Six U-Pb and Sm-Nd analyses in granitoid rocks of the Cachoeirinha suite yielded ages of 1587-1522 Ma and $\mathrm{T}_{\mathrm{DM}}$ model ages of 1.88-1.75 Ga (Epsilon $_{\mathrm{Nd}}$ values of -0.8 to +1.0 ). In addition, three post-tectonic plutonic rocks yielded $\mathrm{U}-\mathrm{Pb}$ ages from $1485-1389 \mathrm{Ma}$ ( $\mathrm{T}_{\mathrm{DM}}$ of $1.77-1.74 \mathrm{Ga}$ and Epsilon ${ }_{\mathrm{Nd}}$ values from -1.3 to +1.7 ). Variations in major and trace elements of the Cachoeirinha suite rocks indicate fractional crystallization process and magmatic arc geologic setting. These results suggest the following interpretations: (1) The interval of 1590-1520 Ma represents an important magmatic activity in SW Amazonian craton. (2) $\mathrm{T}_{\mathrm{DM}}$ and arc-related chemical affinity support the hypothesis that the rocks are genetically associated with an east-dipping subduction zone under the older (1.79-1.74 Ga) continental margin. (3) The 1590-1520 Ma age of intrusive rocks adjacent to an older crust represents similar geological framework along the southern margin of Baltica, corroborating the hypothesis of tectonic relationship at that time.
\end{abstract}

Key words: Amazonian craton, $\mathrm{U}-\mathrm{Pb}$ and $\mathrm{Sm}-\mathrm{Nd}$ geochronology, Mesoproterozoic, juvenile magmatism.

\section{INTRODUCTION}

The Amazonian craton represents a key region for reconstruction of Meso- and Paleoproterozoic continents. To understand its geological framework and crustal evolution is, therefore, essential for such re-

* Member Academia Brasileira de Ciências Correspondence to: Prof. Dr. Mauro Cesar Geraldes E-mail: geraldes@uerj.br construction models. Hoffman (1991) and Dalziel (1992, 1997) suggested that Amazonia amalgamated with Laurentia-Baltica to form Rodinia supercontinent as a result of the worldwide 1.1 to $1.0 \mathrm{Ga}$ melting event. Others authors have also interpreted Amazonia as the continent which collided with Laurentia-Baltica (e.g. Moores 1991, Unrug 1996) at that time. 
Alternatively, Sadowsky and Bettencourt (1996) suggested that Amazonia was already joined to Laurentia-Baltica at $1.6 \mathrm{Ga}$. However, Amazonia became an isolated continental mass during the Mesoproterozoic rifting (probably correlated to the break-up of the Columbia supercontinent, according to Rogers (1996). This extensional event is probably coeval with the Pinwarian orogeny (1.51$1.45 \mathrm{Ga}$ ), reported by Gower and Tucker (1994) in the Grenville Province, followed by a complete Wilson cycle which led to further agglutination to Laurentia-Baltica and formation of Rodinia. There is a lack of paleomagnetic information in Amazonia between $1.6 \mathrm{Ga}$ and $1.4 \mathrm{Ga}$. However, recent paleomagnetic data (Tohver et al. 2002) support juxtaposition of SW Amazonia with Laurentia-Baltica at ca. $1200 \mathrm{Ma}$ and D’Agrella et al. (2000) reported paleomagnetic data indicating another collision at $1000 \mathrm{Ma}$.

The ages, structures, and compositions of the rock units and orogenic events within Amazonia are not well known, in spite of significant recent studies (Bettencourt et al. 1999, Tassinari et al. 2000, Geraldes et al. 2001). In order to improve the knowledge of the SW portion of the Amazonian craton, this paper presents new U-Pb ages, $\mathrm{Sm}-\mathrm{Nd}$ isotopic results and chemical data on granitoids constraining the nature of the Mesoproterozoic plutonic rocks that crop out in SW Mato Grosso State (Brazil). Furthermore, the implications for the Mesoproterozoic crustal formation in SW Amazonian craton are discussed providing potential tectonic correlation between Amazonia and LaurentiaBaltica at 1.59-1.52 Ga.

\section{THE AMAZONIAN CRATON}

The Amazonian craton consists of five NWSE trending Proterozoic provinces that developed adjacent to an Archean core (Teixeira et al. 1989, Tassinari and Macambira 1999, Santos et al. 2000). Recent U-Pb geochronology supported by Nd isotopic mapping defined the 2.25-2.00 Ga MaroniItacaiúnas Province (MIP) and the 2.00-1.80 Ga
Ventuari-Tapajós Province (VTP) (Tassinari et al. 1997, 2000, Tassinari and Macambira 1999). The slightly younger Rio Negro-Juruena Province (RNJP) is dominated by granitic, granodioritic, tonalitic gneisses and migmatites with crystallization ages varying from $1.80 \mathrm{Ga}$ to $1.63 \mathrm{Ga}$ and $\mathrm{Pb}$, $\mathrm{Nd}$ and $\mathrm{Sr}$ isotopic data also indicate that it is a juvenile accretionary province (Tassinari et al. 1997, 2000, Tassinari and Macambira 1999, Geraldes et al. 2001). VTP rocks are locally overlain by ca. 1.8$1.7 \mathrm{Ga}$ felsic to intermediate volcanic rocks (Neder et al. 2002).

The Rondonian-San Ignácio Province (RSIP) is parallel to and outboard of the RNJP and consists of accretionary juvenile material formed from $1.58 \mathrm{Ga}$ to $1.36 \mathrm{Ga}$ (Tassinari et al. 2000, Tassinari and Macambira 1999, Geraldes et al. 2001) strongly affected and reworked by metamorphic events at about $1.34 \mathrm{Ga}$ (Tassinari et al. 2000), 1.20 Ga (Tohver et al. 2002), and $1.11 \mathrm{Ga}$ (Rizzotto, personal communication). Several suites of 1.6 to $1.0 \mathrm{Ga}$ undeformed plutons with rapakivi characteristics intrude the RNJP. This spectrum could be grouped in 6 periods: $1.61-1.53 \mathrm{Ga}, 1.41 \mathrm{Ga}, 1.39 \mathrm{Ga}, 1.35-1.31 \mathrm{Ga}$, 1.31-1.30 Ga, 1.0-1.08 Ga (Bettencourt et al. 1999).

Alternatively, Santos et al. (2000) suggested a new configuration for the geochronological provinces of SW Amazonian craton. In this proposition, Santos et al. (2000) renamed the Rio Negro-Juruena, suggesting the name Rio Negro for the rocks above the Amazonas river, and Juruena-Rondonian for the Rio Negro-Juruena sensu Tassinari and Macambira (1999). In this paper we use the SW Amazonian craton division according to Tassinari and Macambira (1999).

The ca. 1.0 Ga Sunsás Province occurs west of the Rondonian/San Ignacio Province in Bolivia (Litherland et al. 1989) and includes the Sunsás and Aguapeí groups, comprised of low-grade to undeformed supracrustal sequences, and the high grade metamorphic rocks of the Nova Brasilândia Group (Rizzotto, personal communication). These sedimentary rocks locally overlie the basement of the Rio Negro/Juruena and Rondônia-San Ignacio Pro- 
TABLE I

A time-correlation table showing the synthesis of the major juvenile accretionary events, anorogenic plutonism and sedimentation of the SW Amazonian craton in Mato Grosso state.

\begin{tabular}{|c|c|c|c|c|}
\hline \multicolumn{2}{|c|}{ Rio Negro/Juruena Province } & \multicolumn{2}{|c|}{ Rondonian/San Ignácio Province } & \multirow{2}{*}{$\begin{array}{c}\text { Sunsás Province } \\
\text { Aguapeí Thrust }\end{array}$} \\
\hline $\begin{array}{l}\text { Alto Jauru } \\
\text { orogen }\end{array}$ & $\begin{array}{c}\text { Cachoeirinha } \\
\text { orogen }\end{array}$ & $\begin{array}{l}\text { Rio Alegre } \\
\text { orogen }\end{array}$ & $\begin{array}{l}\text { Santa Helena } \\
\text { orogen }\end{array}$ & \\
\hline $\begin{array}{l}\text { Tonalites and } \\
\text { granites formed } \\
\text { between 1.79-1.75 } \\
\text { Ga, in arc-related } \\
\text { tectonic setting }\end{array}$ & $\begin{array}{l}\text { Tonalites and } \\
\text { granites formed } \\
\text { between } 1.59-1.52 \\
\text { Ga, in arc-related } \\
\text { tectonic setting }\end{array}$ & $\begin{array}{l}\text { MORB, BIF and } \\
\text { cherts ( } 1.51 \mathrm{Ga}) \\
\text { Tonalites and } \\
\text { granites formed } \\
\text { between } 1.79-1.75 \\
\text { Ga, in island arc } \\
\text { tectonic setting }\end{array}$ & & \\
\hline $\begin{array}{l}\text { Intracontinental } \\
\text { magmatism, } \\
\text { (rapakivi granites } \\
\text { and related } \\
\text { mafic roks) } \\
1.47-1.43 \mathrm{Ga}\end{array}$ & & & $\begin{array}{l}\text { Granodiorites and } \\
\text { granites formaded } \\
\text { between } 1.45-1.42 \\
\text { Ga, in arc-related } \\
\text { tectonic setting }\end{array}$ & \\
\hline $\begin{array}{l}\text { Aguapeí Group } \\
\text { deposition } \\
(<1.1 \mathrm{Ga})\end{array}$ & $\begin{array}{l}\text { Aguapeí Group } \\
\text { deposition } \\
(<1.1 \mathrm{Ga})\end{array}$ & $\begin{array}{l}\text { Aguapeí Group } \\
\text { deposition } \\
(<1.1 \mathrm{Ga})\end{array}$ & $\begin{array}{l}\text { Aguapeí Group } \\
\text { deposition } \\
(<1.1 \mathrm{Ga})\end{array}$ & $\begin{array}{l}\text { Aguapeí Group } \\
\text { deposition } \\
(<1.1 \mathrm{Ga})\end{array}$ \\
\hline
\end{tabular}

vinces (Table I). The tectonic event between 970$840 \mathrm{Ma}$ in the Aguapeí thrust belt in SW Mato Grosso (Geraldes et al. 1997) is apparently coeval with that identified in the Sunsás Province, and some authors (Tassinari and Macambira 1999) have interpreted the Aguapeí thrust belt as a branch of the Sunsás deformation front reported in Bolivia (Litherland et al. 1986).

\section{GEOLOGY OF SW MATO GROSSO STATE}

Three major geochronological and tectonic provinces (Teixeira et. al. 1989, Tassinari and Macambira 1999) are traditionally considered and represented in the western part of the Amazonian craton in Mato Grosso state: Rio Negro-Juruena Province, Rondonian-San Ignacio Province, and Sunsás Province. 
Recent advances in understanding the evolution of these provinces, based on newly achieved geochronological and geological data (Tassinari et al. 1997, 2000, Sato and Tassinari 1997, Pinho et al. 1997, Bettencourt et al. 1999) among others, provide the basis to the better understanding of these provinces. In this way the geochronological provinces can be subdivided in various orogenies and terranes, which have evolved within the timeperiod established for each province.

The Proterozoic basement in SW Mato Grosso (Figure 1) consists of igneous and metamorphic rocks interpreted as belonging to the RNJP. This province includes distinct rock associations, such as the oldest Alto Jauru orogenic rocks (Pinho et al. 1997) encompassing acid metavolcanics associated to BIF's and metasedimentary rocks and tonalitic to granitic gneisses. U-Pb ages (volcanic and plutonic rocks) vary from 1790 to $1740 \mathrm{Ma}\left(\varepsilon_{\mathrm{Nd}}\right.$ from +2.6 to +2.2 and $\mathrm{T}_{\mathrm{DM}}$ from 2.00 to $1.80 \mathrm{Ga}$ ) interpreted as originated in a volcanic arc due to their juvenile isotopic signatures and chemical characteristics (Pinho et al. 1997, Geraldes et al. 2001).

The geological map presented in Figure 1 is compiled from (unpublished data) and Carneiro et al. (1992) and shows intrusive rocks in the Alto Jauru greenstone belt rocks. These intrusive rocks are here named Cachoeirinha suite and petrologic, field evidences, $\mathrm{U}-\mathrm{Pb}$ and $\mathrm{Sm}-\mathrm{Nd}$ geochronology of these rocks are the objectives of this work.

In the eastern sector of the studied area occurs the Rio Branco suite, composed of bimodal intrusive rocks (rapakivi granites and alkaline basalts) dated about 1.47-1.42 Ga (unpublished data). The youngest unit in the studied area (Figure 1) is represented by the Aguapeí Group, which is composed by slightly metamorphosed claystones, sandstones and conglomerates. These sedimentary rocks were probably deposited between $1.3 \mathrm{Ga}$ to $1.0 \mathrm{Ga}$, and cover all the units previously described. In addition, the rocks in the western boundary of the RNJP are ascribed to the Rondonian-San Ignácio Province, where three orogenic belts have been described. These are the Santa Helena, Rio Alegre (in Brazil) and San Ignácio (in Bolivia). Several granites occur in the west of the studied area such as the large batholith defined as 1.45-1.42 Ga Santa Helena orogen (Geraldes et al. 2001). The 1.42-1.36 Ga San Ignacio orogen (Geraldes et al. 2002) and the 1.501.48 Ga Rio Alegre orogen (Matos et al. 2004) may be interpreted as accretionary arcs related of the SW Amazonian craton crust formation during the Mesoproterozoic.

\section{ANALYTICAL PROCEDURES}

Major element analyses were carried out at the Geochemistry Laboratory, Department of Mineralogy and Geotectonics of the University of São Paulo, using an ICO-ES according to the procedures described in Janasi et al. (1996). Trace element including REE were analyzed at the ACTLAB (Toronto, Canada) using ICP-MS routine.

For the U-Pb analyses 20 to $30 \mathrm{~kg}$ of sample were crushed, milled and heavy minerals were concentrated in wiffley table at University of São Paulo (Brazil). Heavy liquids were used for separation of zircon. U-Pb zircon analyses were carried out in the Isotope Geochemistry Laboratory (IGL), Department of Geology, University of Kansas (USA). The less magnetic fraction was abraded and handpicked single grains were spiked with ${ }^{205} \mathrm{~Pb}^{235} \mathrm{U}$ mixed tracer. Zircons were dissolved and $\mathrm{Pb}$ and U were separated using procedures modified after Krogh (1973, 1982) and Parrish (1987). Zircon weight varied from 0.001 to $0.005 \mathrm{mg}$. Isotopic ratios were measured using a VG Sector multicollector mass spectrometer in a single collector mode using Daly detector.

$\mathrm{Pb}$ isotope compositions were analyzed on single Re filaments using silica gel and phosphoric acid. Uranium was loaded with $\mathrm{Pb}$ on the same filament and analyzed as $\mathrm{UO}_{2}^{+}$. Radiogenic ${ }^{208} \mathrm{~Pb},{ }^{207} \mathrm{~Pb}$, and ${ }^{206} \mathrm{~Pb}$ were calculated by correcting for laboratory $\mathrm{Pb}$ blank (from 55 to $17 \mathrm{pg}$ of total $\mathrm{Pb}$ during the analyses) and for nonradiogenic common $\mathrm{Pb}$ corresponding to Stacey and Kramers (1975) model for approximate age of the sample. Decay con- 


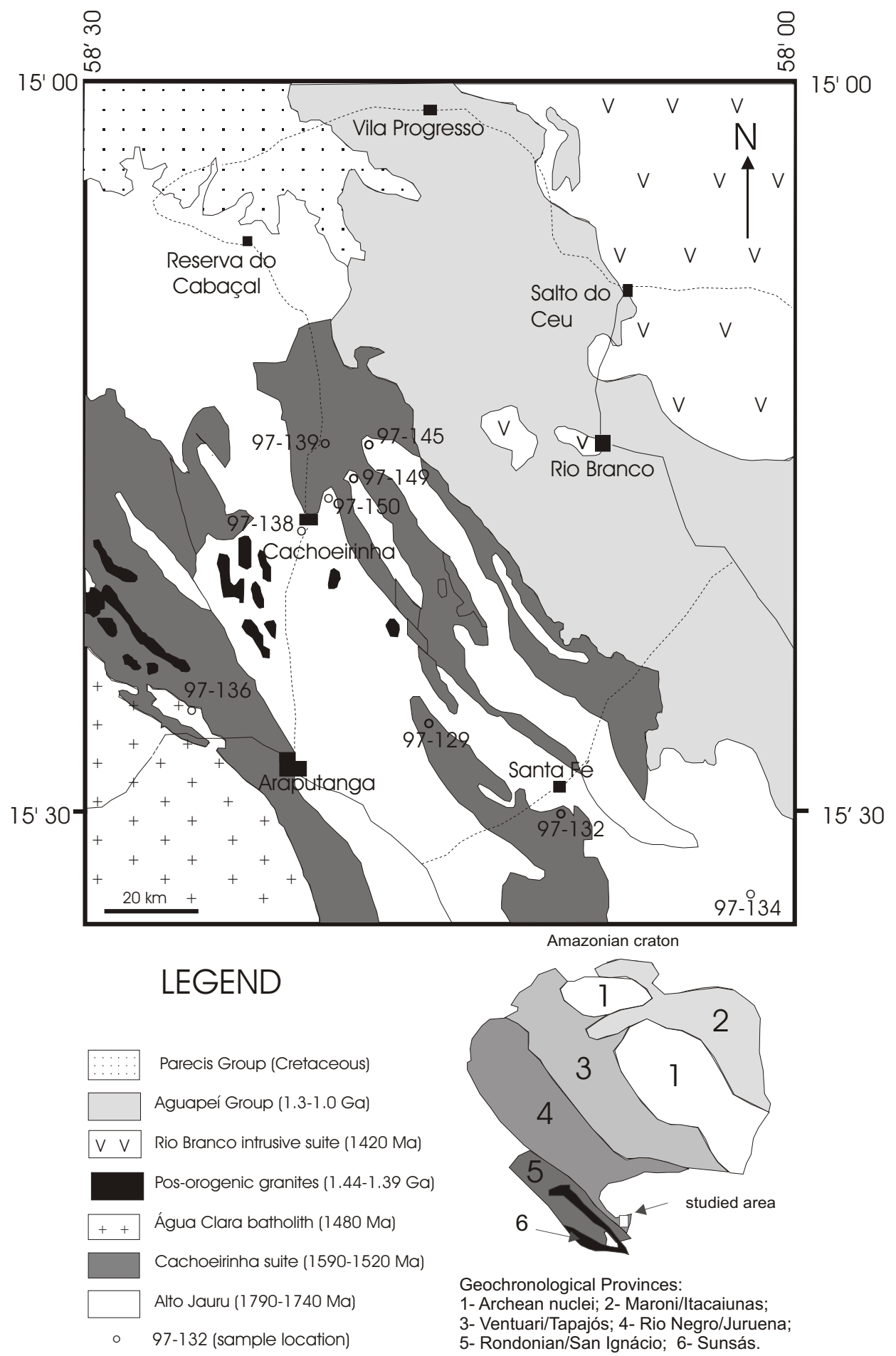

Fig. 1 - Geological map of the studied area, SW Amazon Craton, Mato Grosso State, Brazil. (samples 97-130, 97-134, 97-145, 97-149 and 97-150 are too small bodies within Alto Jauru terrane to be displayed at the map scale). 
stants used were $0.155125 \times 10^{-9}$ year $^{-1}$ for ${ }^{238} \mathrm{U}$ and $0.98485 \times 10^{-9}$ year $^{-1}$ for ${ }^{235} \mathrm{U}$ (Steiger and Jäger 1977). Zircon data were regressed using the ISOPLOT program of Ludwig (1998). Uncertainties on concordia intercept ages are given at the 2 sigma $(\sigma)$ level.

For the Sm-Nd analyses, rock powders were dissolved in bombs at ca. $180^{\circ} \mathrm{C}$ and spiked with ${ }^{145} \mathrm{Nd}$ and ${ }^{144} \mathrm{Sm}$. REE were extracted using the methodology of Patchett and Ruiz (1987). Isotopic compositions were measured in a VG Sector 5collectors mass spectrometer. Sm was loaded with $\mathrm{H}_{3} \mathrm{PO}_{4}$ on a single Ta filament and typically analyzed as $\mathrm{Sm}^{+}$in a static multicolector mode. Nd was loaded with phosphoric acid on a single Re filament having a thin layer of AGW-50 resin beads and analyzed as $\mathrm{Nd}^{+}$using static mode. Analyses of BCR-1 during the period when unknown samples were analyzed yielded $\mathrm{Nd}=29.44 \pm 0.70 \mathrm{ppm}, \mathrm{Sm}=6.77 \pm$ $0.21 \mathrm{ppm},{ }^{147} \mathrm{Sm} /{ }^{144} \mathrm{Nd}=0.13931 \pm 0.00071$, and ${ }^{143} \mathrm{Nd} /{ }^{144} \mathrm{Nd}=0.512641 \pm 0.000007$, yielding $\varepsilon_{\mathrm{Nd}}$ $=0.07 \pm 0.12($ all at $1 \sigma)$. Sm-Nd model ages $\left(\mathrm{T}_{\mathrm{DM}}\right)$ were calculated according to DePaolo (1988). During the course of these analyses Nd blanks ranged from 500 to $150 \mathrm{pg}$, with corresponding Sm blanks of 100 to $50 \mathrm{pg}$. Correction for blanks was insignificant for $\mathrm{Nd}$ isotopic composition and insignificant for Sm-Nd concentrations and ratios. Sm-Nd ratios are corrected to within \pm 0.5 percent based on analytical uncertainties.

\section{U-Pb AND Sm-Nd RESULTS}

Nine rocks of the studied area (Figure 1) were analyzed for U-Pb and Sm-Nd (summarized in Table II). Complete U-Pb results are presented in Table III and Sm-Nd results in Table IV. Chemical results from 9 samples of studied area are presented in Table V.

The Cachoeirinha suite rocks show compositional variations from tonalites, granodiorites to granites. These rocks are leucocratic, gray to green in color and medium-grained size ( 0.1 to $1 \mathrm{~cm}$ ), presenting isotropic, slightly foliated and banded fabrics. The essential mineralogy includes plagioclase, amphibole, biotite, quartz and K-feldspar. Zircon, apatite, allanite and oxides are the accessory minerals.

Several plutonic bodies were individualized from the gneissic basement in the Cachoeirinha region according to the geologic mapping published by Saes et al. (personal communication), (unpublished data) and Carneiro et al. (1992). The authors above cited worked in different locations, what hampered the temporal relationship between the granitoid intrusions observed in the respective studied areas. However, the nomenclature described by these authors was used during the sampling work and include the following granitoids: Quatro Marcos, $\mathrm{Ca}-$ choeirinha, Santa Cruz, Alvorada and Água Clara. The new units sampled and analyzed in this paper without previous studies were described using new denominations as Santa Fé, São Domingos and Araputanga.

\section{Quatro Marcos Tonalite}

Two tonalitic rocks were sampled for U-Pb dating. The first one (sample 97-134) was collected in the southeastern region of the Santa Fé town. In this region Carneiro et al. (1992) identified Paleoproterozoic (1.9-1.7 Ga) $\mathrm{Rb}$-Sr ages on a gray gneiss. Four zircons were analyzed for $\mathrm{U}-\mathrm{Pb}$ from a gray tonalitic gneiss and yielded an upper intercept age of $1536 \pm 11 \mathrm{Ma}$ with $\mathrm{T}_{\mathrm{DM}}=1.77$ and $\varepsilon_{\mathrm{Nd}}=+0.5$ (Figure 2A; Table II).

\section{Chachoeirinha Tonalite}

The second tonalitic sample (97-150) was collected $2 \mathrm{~km}$ to the west of Cachoeirinha town (Figure 1). Zircons obtained from this rock yielded upper intercept U-Pb age (Figure 2B; Table II) of $1549 \pm 10 \mathrm{Ma}$ and whole rock $\mathrm{Sm}-\mathrm{Nd}$ analysis yielded $\mathrm{T}_{\mathrm{DM}}=1.83$ and $\varepsilon_{\mathrm{Nd}}=+1.0$.

\section{SÃo Domingos GNeISS}

This sample (97-149) presents a compositional banding of quartz and feldspar, in felsic layers, and biotite and amphibole-enriched in mafic layers. The São Domingos gneiss yielded an U-Pb zircon 
TABLE II

$\mathrm{U}-\mathrm{Pb}$ ages and $\mathrm{Sm}-\mathrm{Nd}$ isotopic properties of rocks from Cachoeirinha suite.

\begin{tabular}{l|l|c|c|c|c|c}
\hline Sample & Lithotype & $\mathrm{U}-\mathrm{Pb}(\mathrm{Ma})$ & $\varepsilon_{\mathrm{Nd}(0)}$ & $\varepsilon_{\mathrm{Nd}(\mathrm{t})}$ & $\mathrm{T}_{\mathrm{DM}}$ & $f$ \\
\hline $97-134$ & Quatro Marcos Tonalite & $1536 \pm 11$ & -14.2 & +0.5 & 1.77 & -0.38 \\
$97-159$ & Cachoeirinha Tonalite & $1549 \pm 10$ & -14.7 & +1.0 & 1.83 & -0.40 \\
$97-149$ & São Domingos Gneiss & $1587 \pm 04$ & -15.0 & -0.8 & 2.05 & -0.36 \\
$97-138$ & Granite Cachoeirinha & $1522 \pm 12$ & -19.6 & +0.9 & 1.78 & -0.54 \\
$97-132$ & Santa Fé Granite & $1537 \pm 06$ & -22.2 & +0.5 & 1.75 & -0.60 \\
$97-145$ & Santa Cruz Gneiss & $1562 \pm 36$ & -20.2 & +0.9 & 1.79 & -0.53 \\
$97-136$ & Água Clara Granodiorite & $1485 \pm 04$ & -5.0 & +1.7 & 1.77 & -0.50 \\
$97-139$ & Araputanga Granite & $1440 \pm 06$ & -20.2 & -0.2 & 1.74 & -0.56 \\
$97-129$ & Alvorada Granite & $1389 \pm 03$ & -20.3 & -1.3 & 1.77 & -0.54 \\
\hline
\end{tabular}

age (Figure 2C; Table II) in the range of 1587 to $1489 \mathrm{Ma}$ and $\mathrm{T}_{\mathrm{DM}}=2.05 \mathrm{Ga}$ and $\varepsilon_{\mathrm{Nd}(1489)}=-0.8$. The widespread distribution of the results when plotted in the concordia diagram also suggest the presence of inherited zircons which upper intercept indicate the age about $1.7 \mathrm{Ga}$.

\section{SANTA Cruz GneISS}

This granodioritic rock (sample 97-145) is surrounded by the São Domingos gneiss and may comprise lateral variations (unpublished data) of the last unit. The Santa Cruz sample yielded U-Pb zircon age (Figure 2D; Table II) of $1562 \pm 36$, and $\mathrm{T}_{\mathrm{DM}}$ age of $1.79 \mathrm{Ga}$ and $\varepsilon_{\mathrm{Nd}(1562)}=+0.9$.

\section{Cachoeirinha Granite}

The analyzed sample (97-138) was collected from a plutonic body intruded in the São Domingos Gneiss (sample 97-149). The upper intercept yielded U-Pb zircon age of $1522 \pm 11 \mathrm{Ma}, \mathrm{T}_{\mathrm{DM}}=1.78 \mathrm{Ga}$ and $\varepsilon_{\mathrm{Nd}(1522)}=+0.9$ (Figure 2E; Table II).

\section{SAnta Fé Granite}

Isotropic granitic rocks related to the Cachoeirinha suite are widespread within the Santa Fé-Araputanga region (Figure 1). The Santa Fé granite (sample 97-132) yielded U-Pb zircon ages of $1546 \pm 15 \mathrm{Ma}$ and $\mathrm{T}_{\mathrm{DM}}=1.78 \mathrm{Ga}$ and $\varepsilon_{\mathrm{Nd}(1537)}=+0.9$ (Figure $2 \mathrm{~F}$; Table II).
In addition, three post-orogenic plutons of granitic and granodioritic compositions reported in previous studies (Saes, Monteiro and Matos personal communication) were analyzed. The Água Clara is described as a large anorogenic batholith in the Jauru region $\left(600 \mathrm{~km}^{2}\right)$ and comprises mainly granodiorites with subordinate granites. The Alvorada granite comprises several isotropic granitic bodies widespread within the Jauru-Araptanga region. Araputanga granite is a small body intrusive in banded gneisses in Araputanga region.

\section{Água Clara Granodiorite}

$\mathrm{U}-\mathrm{Pb}$ zircon analysis yielded $1468 \pm 35 \mathrm{Ma}$ (sample 97-136, Figure 3A; Table II), with the $\mathrm{T}_{\mathrm{DM}}$ age is $1.77 \mathrm{Ga}$ and $\varepsilon_{\mathrm{Nd}(1485)}=+1.7$.

\section{Alvorada Granite}

The 97-129 sample yielded two discordia lines, indicating U-Pb zircon ages (Figure 3B; Table II) of $1483 \pm 10 \mathrm{Ma}$ and $1440 \pm 06 \mathrm{Ma}\left(\mathrm{T}_{\mathrm{DM}}=1.74 \mathrm{Ga}\right.$ and $\left.\varepsilon_{\mathrm{Nd}(1440)}=-0.2\right)$.

\section{Araputanga Granite}

These granitic bodies are interpreted (Pinho et al. 1997 among others) as the youngest magmatic event in the region. The 97-139 sample yielded U$\mathrm{Pb}$ zircon age (Figure 3C; Table II) of $1394 \pm 37 \mathrm{Ma}$ $\left(\mathrm{T}_{\mathrm{DM}}=1.77 \mathrm{Ga}\right.$ and $\left.\varepsilon_{\mathrm{Nd}(1440)}=-1.3\right)$. 
TABLE III

U-Pb isotope data from Cachoeirinha suite rocks.

\begin{tabular}{|c|c|c|c|c|c|c|c|c|c|c|c|}
\hline \multirow[t]{2}{*}{ Fraction } & \multirow{2}{*}{$\begin{array}{l}\text { Size } \\
(\mathrm{mg})\end{array}$} & \multirow{2}{*}{$\begin{array}{c}\mathrm{U} \\
(\mathrm{ppm})\end{array}$} & \multirow{2}{*}{$\begin{array}{c}\mathrm{Pb} \\
(\mathrm{ppm})\end{array}$} & \multicolumn{3}{|c|}{ Observed \# } & \multirow{2}{*}{$\begin{array}{c}\text { Radiogenic } \\
\text { Ratios } \dagger \\
{ }^{207} \mathrm{~Pb} /{ }^{206} \mathrm{~Pb}\end{array}$} & \multirow[b]{2}{*}{ (Rho) } & \multicolumn{3}{|c|}{ Calculated Ages $\pm 2 \mathrm{SE}(\mathrm{Ma}) \ddagger$} \\
\hline & & & & $\begin{array}{l}{ }^{206} \mathrm{~Pb} / \\
{ }^{204} \mathrm{~Pb}\end{array}$ & $\begin{array}{c}{ }^{207} \mathrm{~Pb} / \\
{ }^{235} \mathrm{U}\end{array}$ & $\begin{array}{c}{ }^{206} \mathrm{~Pb} / \\
{ }^{238} \mathrm{U}\end{array}$ & & & ${ }^{207} \mathrm{~Pb} /{ }^{235} \mathrm{U}$ & ${ }^{206} \mathrm{~Pb} /{ }^{238} \mathrm{U}$ & 207/206 \\
\hline \multicolumn{12}{|l|}{$97-129$} \\
\hline \multicolumn{12}{|c|}{ Alvorada Granite } \\
\hline $\mathrm{NM}(3)[1]$ & 0.003 & 866 & 240 & 431 & 2.93909 & 0.24140 & 0.088302 & 0.976 & $1393 \pm 10$ & $1394 \pm 09$ & $1389 \pm 2.9$ \\
\hline $\mathrm{M}(3)[1] \mathrm{a}$ & 0.005 & 2860 & 403 & 1355 & 1.52602 & 0.13619 & 0.081255 & 0.985 & $941 \pm 05$ & $823 \pm 04$ & $1228 \pm 1.7$ \\
\hline $\mathrm{M}(4)[1]$ & 0.004 & 2219 & 267 & 1111 & 1.27879 & 0.11257 & 0.082391 & 0.981 & $836 \pm 05$ & $688 \pm 03$ & $1255 \pm 2.1$ \\
\hline $\mathrm{NM}(3) \mathrm{b}$ & 0.002 & 1356 & 261 & 624 & 2.16566 & 0.17780 & 0.088336 & 0.965 & $1170 \pm 09$ & $1055 \pm 07$ & $1390 \pm 3.8$ \\
\hline $\mathrm{NM}(3) \mathrm{b}$ & 0.002 & 594 & 148 & 194 & 2.24644 & 0.18656 & 0.087329 & 0.917 & $119 \pm 16$ & $1103 \pm 13$ & $1368 \pm 11$ \\
\hline \multicolumn{12}{|l|}{ 97-132 } \\
\hline \multicolumn{12}{|c|}{ Santa Fé Granite } \\
\hline $\mathrm{NM}(5)[1]$ & 0.001 & 1327 & 239 & 602 & 1.92455 & 0.16038 & 0.087030 & 0.982 & $1090 \pm 10$ & $959 \pm 10$ & $1361 \pm 3.2$ \\
\hline $\mathrm{M}(6)[1]$ & 0.002 & 1553 & 318 & 1516 & 2.38753 & 0.19195 & 0.090209 & 0.984 & $1239 \pm 09$ & $1132 \pm 08$ & $1430 \pm 2.5$ \\
\hline NM(5) "O" & 0.002 & 1348 & 323 & 3873 & 3.24699 & 0.24842 & 0.094794 & 0.985 & $1469 \pm 12$ & $1430 \pm 11$ & $1524 \pm 2.6$ \\
\hline \multicolumn{12}{|l|}{ 97-134 } \\
\hline \multicolumn{12}{|c|}{ Quatro Marcos Tonalite } \\
\hline $\mathrm{NM}(-1)[1]$ & 0.007 & 1013 & 256 & 3749 & 3.09600 & 0.23424 & 0.095860 & 0.992 & $1432 \pm 07$ & $1357 \pm 07$ & $1545 \pm 12$ \\
\hline $\mathrm{M}(1)[1]$ & 0.006 & 802 & 184 & 2667 & 2.80773 & 0.21244 & 0.095855 & 0.991 & $1358 \pm 07$ & $1242 \pm 07$ & $1545 \pm 1.4$ \\
\hline $\mathrm{M}(-1)[1]$ & 0.009 & 977 & 276 & 3601 & 3.44264 & 0.26153 & 0.095469 & 0.993 & $1514 \pm 08$ & $1498 \pm 08$ & $1537 \pm 1.1$ \\
\hline $\mathrm{M}(0)[1]$ & 0.002 & 688 & 148 & 1595 & 2.61705 & 0.19701 & 0.096344 & 0.991 & $1305 \pm 07$ & $1159 \pm 06$ & $1555 \pm 1.2$ \\
\hline \multicolumn{12}{|l|}{$97-136$} \\
\hline \multicolumn{12}{|c|}{ Agua Clara Granodiorite } \\
\hline $\mathrm{NM}(0)[1]$ & 0.007 & 632 & 139 & 1099 & 2.73623 & 0.21152 & 0.093818 & 0.979 & $1338 \pm 07$ & $1237 \pm 06$ & $1505 \pm 1.8$ \\
\hline $\mathrm{M}(0)[1]$ & 0.004 & 395 & 85 & 1824 & 3.00620 & 0.21321 & 0.096798 & 0.965 & $1368 \pm 08$ & $1246 \pm 07$ & $1563 \pm 2.7$ \\
\hline $\mathrm{M}(1)[1]$ & 0.007 & 479 & 83 & 3144 & 2.29866 & 0.17573 & 0.094871 & 0.962 & $1212 \pm 07$ & $1044 \pm 06$ & $1526 \pm 3.0$ \\
\hline NM (0) & 0.004 & 799 & 192 & 674 & 2.95776 & 0.30399 & 0.093310 & 0.953 & $1396 \pm 12$ & $1337 \pm 11$ & $1490 \pm 5.0$ \\
\hline NM (0) & 0.004 & 62 & 22 & 106 & 3.06905 & 0.242033 & 0.091966 & 0.969 & $1425 \pm 57$ & $1397 \pm 54$ & $1467 \pm 19$ \\
\hline \multicolumn{12}{|l|}{ 97-138 } \\
\hline \multicolumn{12}{|c|}{ Cachoeirinha Granite } \\
\hline $\mathrm{NM}(0)[2]$ & 0.008 & 161 & 51 & 411 & 3.50167 & 0.26626 & 0.095379 & 0.970 & $1528 \pm 13$ & $1522 \pm 13$ & $1536 \pm 3.9$ \\
\hline $\mathrm{M}(0)[2]$ & 0.011 & 668 & 150 & 702 & 2.01803 & 0.17121 & 0.085486 & 0.961 & $1121 \pm 06$ & $1019 \pm 08$ & $1327 \pm 2.8$ \\
\hline $\mathrm{M}(1)[2]$ & 0.004 & 1156 & 284 & 1316 & 2.90693 & 0.22359 & 0.094290 & 0.956 & $1384 \pm 07$ & $1301 \pm 07$ & $1514 \pm 03$ \\
\hline $\mathrm{M}(2)[2]$ & 0.004 & 2103 & 430 & 1191 & 2.27294 & 0.18208 & 0.090533 & 0.865 & $1204 \pm 12$ & $1078 \pm 12$ & $1437 \pm 13$ \\
\hline \multicolumn{12}{|l|}{ 97-139 } \\
\hline \multicolumn{12}{|c|}{ Araputanga Granite } \\
\hline NM(5)"M" [1] & 0.002 & 139 & 39 & 294 & 3.10414 & 0.23967 & 0.093934 & 0.989 & $1434 \pm 28$ & $1385 \pm 27$ & $1507 \pm 5.6$ \\
\hline NM(5) "N" [1] & 0.006 & 2118 & 504 & 417 & 2.43338 & 0.19804 & 0.089116 & 0.969 & $1253 \pm 06$ & $1165 \pm 06$ & $1407 \pm 2.4$ \\
\hline NM(5) "O" [1] & 0.005 & 1035 & 283 & 514 & 2.93237 & 0.23548 & 0.090315 & 0.970 & $1390 \pm 11$ & $1363 \pm 10$ & $1432 \pm 3.7$ \\
\hline NM (5) "F" & 0.010 & 7664 & 1655 & 1945 & 2.83983 & 0.21508 & 0.095758 & 0.991 & $1366 \pm 07$ & $1256 \pm 07$ & $1543 \pm 1.4$ \\
\hline \multicolumn{12}{|l|}{ 97-145 } \\
\hline Santa Cruz Gn & & & & & & & & & & & \\
\hline $\mathrm{M}(-1)[1]$ & 0.004 & 1262 & 329 & 322 & 2.83861 & 0.21308 & 0.096619 & 0.869 & $1366 \pm 15$ & $1245 \pm 12$ & $1560 \pm 10$ \\
\hline $\mathrm{M}(1)[1]$ & 0.013 & 262 & 74 & 956 & 3.44136 & 0.25935 & 0.096236 & 0.991 & $1514 \pm 13$ & $1487 \pm 13$ & $1552 \pm 2.3$ \\
\hline $\mathrm{M}(2)[1]$ & 0.004 & 807 & 180 & 855 & 2.74331 & 0.20886 & 0.095260 & 0.984 & $1340 \pm 14$ & $1223 \pm 12$ & $1533 \pm 3.4$ \\
\hline
\end{tabular}

\section{GEOCHEMISTRY}

The major and trace elements analyses $(n=9$, Table $\mathrm{V}$ ) of tonalites, granodiorites and granites of $\mathrm{Ca}$ choeirinha suite show variation content in compatible elements related to a calc-alkaline fractional differentiation. The $\mathrm{SiO}_{2}$ amount ranges from $44 \%$ to $55 \%$ in the more primitive rocks, $67 \%$ to $68 \%$ in the intermediate rocks, and reaches 70 to $73 \%$ in the more fractionated rocks. $\mathrm{CaO}$ ranges from $7 \%$ to $8 \%, 4 \%$ to $5 \%$, and $2 \%$ to $3 \%$, and $\mathrm{MgO}$ ranges from $4.7 \%$ to $7.2 \%, 1 \%$ to $1.2 \%$, and $0.3 \%$ to $0.8 \%$, respectively in the tonalitic (more primitive), granodioritic (intermediated) and granitic (evolved) rocks. Trace element results plot within the field of volcanic arc granites (VAG), following the Pearce et al. (1984) tectonic discrimination diagram (Figure 4A) for both orogenic and post-orogenic rocks. In 
TABLE III (continuation)

\begin{tabular}{|c|c|c|c|c|c|c|c|c|c|c|c|}
\hline \multirow[t]{2}{*}{ Fraction } & \multirow{2}{*}{$\begin{array}{l}\text { Size } \\
(\mathrm{mg})\end{array}$} & \multirow{2}{*}{$\begin{array}{c}\mathrm{U} \\
(\mathrm{ppm})\end{array}$} & \multirow{2}{*}{$\begin{array}{c}\mathrm{Pb} \\
(\mathrm{ppm})\end{array}$} & \multicolumn{3}{|c|}{ Observed \# } & \multirow{2}{*}{$\begin{array}{c}\text { Radiogenic } \\
\text { Ratios } \dagger \\
{ }^{207} \mathrm{~Pb} /{ }^{206} \mathrm{~Pb}\end{array}$} & \multirow[b]{2}{*}{ (Rho) } & \multicolumn{3}{|c|}{ Calculated Ages $\pm 2 \mathrm{SE}(\mathrm{Ma}) \doteqdot$} \\
\hline & & & & $\begin{array}{l}{ }^{206} \mathrm{~Pb} / \\
{ }^{204} \mathrm{~Pb}\end{array}$ & $\begin{array}{c}{ }^{207} \mathrm{~Pb} / \\
{ }^{235} \mathrm{U}\end{array}$ & $\begin{array}{c}{ }^{206} \mathrm{~Pb} / \\
{ }^{238} \mathrm{U}\end{array}$ & & & ${ }^{207} \mathrm{~Pb} /{ }^{235} \mathrm{U}$ & ${ }^{206} \mathrm{~Pb} /{ }^{238} \mathrm{U}$ & 207/206 \\
\hline \multicolumn{12}{|l|}{ 97-149 } \\
\hline \multicolumn{12}{|c|}{ São Domingos Gneiss } \\
\hline $\mathrm{NM}(-1)[4]$ & 0.006 & 557 & 164 & 544 & 3.69373 & 0.25591 & 0.104681 & 0.970 & $1570 \pm 29$ & $1469 \pm 26$ & $1709 \pm 8.3$ \\
\hline $\mathrm{M}(-1)[4]$ & 0.009 & 469 & 151 & 807 & 4.17118 & 0.28545 & 0.105978 & 0.922 & $1668 \pm 20$ & $1619 \pm 18$ & $1731 \pm 8.6$ \\
\hline $\mathrm{M}(1)[4]$ & 0.008 & 740 & 249 & 439 & 4.07927 & 0.28429 & 0.104067 & 0.903 & $1650 \pm 10$ & $1613 \pm 08$ & $1698 \pm 5.1$ \\
\hline $\mathrm{M}(0)[3]$ & 0.003 & 2134 & 540 & 767 & 2.9358 & 0.22339 & 0.095312 & 0.941 & $1391 \pm 11$ & $1300 \pm 10$ & $1534 \pm 5.3$ \\
\hline $\mathrm{NM}(-1) \mathrm{J}[1]$ & 0.001 & 982 & 130 & 354 & 0.881317 & 0.10496 & 0.060896 & 0.978 & $642 \pm 09$ & $643 \pm 10$ & $636 \pm 7.2$ \\
\hline NM(-1) "K" [1] & 0.001 & 268 & 81 & 337 & 3.81267 & 0.27129 & 0.101927 & 0.964 & $1595 \pm 28$ & $1547 \pm 27$ & $1660 \pm 09$ \\
\hline NM(-1) "L" [1] & 0.001 & 298 & 83 & 510 & 3.68764 & 0.26099 & 0.102474 & 0.990 & $1569 \pm 25$ & $1495 \pm 23$ & $1669 \pm 4.3$ \\
\hline NM(-1) "M " [1] & 0.001 & 339 & 94 & 466 & 3.55433 & 0.25638 & 0.100232 & 0.899 & $153 \pm 29$ & $1471 \pm 24$ & $1628 \pm 15$ \\
\hline NM(-1) "N" [1] & 0.001 & 244 & 87 & 234 & 4.28069 & 0.29589 & 0.104923 & 0.992 & $1690 \pm 33$ & $1671 \pm 33$ & $1713 \pm 4.8$ \\
\hline \multicolumn{12}{|l|}{ 97-159 } \\
\hline \multicolumn{12}{|c|}{ Cachoeirinha Tonalite } \\
\hline $\mathrm{M}(-1)[1]$ & 0.004 & 223 & 76 & 195 & 3.51349 & 0.26562 & 0.095931 & 0.894 & $1530 \pm 21$ & $1519 \pm 18$ & $1546 \pm 12$ \\
\hline $\mathrm{M}(0)[1]$ & 0.002 & 244 & 73 & 262 & 3.46200 & 0.26129 & 0.096093 & 0.981 & $1519 \pm 25$ & $1497 \pm 256$ & $1550 \pm 6.3$ \\
\hline $\mathrm{M}(1)[1]$ & 0.001 & 1312 & 380 & 618 & 3.37969 & 0.25395 & 0.096519 & 0.968 & $1500 \pm 12$ & $1459 \pm 12$ & $1558 \pm 4.1$ \\
\hline $\mathrm{M}(0)$ "b" [1] & 0.002 & 592 & 168 & 1247 & 3.48712 & 0.26401 & 0.095792 & 0.993 & $1524 \pm 12$ & $1510 \pm 12$ & $1544 \pm 1.8$ \\
\hline
\end{tabular}

M, NM refer to magnetic fractions at Franz (at 5 amp). [m] = multigrain; [1] = monocrystal. \#: Not corrected for blank and comum Pb. †: Corrected

for blank and comum lead. $¥$ : Based on constants according to Steiger and Jäger (1977).

TABLE IV

Sm-Nd isotopic results from Cachoeirinha suite rocks.

\begin{tabular}{l|l|c|c|c|c|c|c|c|c|c}
\hline Sample & Lythotype & $\begin{array}{c}\mathrm{Age} \\
\mathrm{U} / \mathrm{Pb}\end{array}$ & $\begin{array}{c}\mathrm{Nd} \\
(\mathrm{ppm})\end{array}$ & $\begin{array}{c}\mathrm{Sm} \\
(\mathrm{ppm})\end{array}$ & $\begin{array}{c}{ }^{147} \mathrm{Sm} / \\
144 \mathrm{Nd}\end{array}$ & $\begin{array}{c}143 \mathrm{Nd} / \\
144 \mathrm{Nd}\end{array}$ & $\begin{array}{c}\mathrm{E}(\mathrm{Nd}) \\
\mathrm{t}=0\end{array}$ & $\begin{array}{c}\mathrm{E}(\mathrm{Nd}) \\
\mathrm{t}(\mathrm{U} / \mathrm{Pb})\end{array}$ & $\begin{array}{c}\mathrm{T}(\mathrm{DM}) \\
\mathrm{Ma}\end{array}$ & $f$ \\
\hline $97-129$ & granite & 1394 & 64.21 & 9.55 & 0.08993 & 0.511595 & -20.34 & -1.27 & 1773 & -0.54 \\
$97-132$ & granite & 1546 & 38.65 & 5.96 & 0.09336 & 0.511632 & -19.62 & 0.87 & 1777 & -0.53 \\
$97-134$ & tonalite & 1536 & 25.88 & 5.24 & 0.12247 & 0.511913 & -14.14 & 0.48 & 1876 & -0.38 \\
$97-136$ & granodiorite & 1468 & 21.61 & 3.48 & 0.09751 & 0.511758 & -17.16 & 1.73 & 1675 & -0.50 \\
$97-138$ & granite & 1521 & 67.67 & 9.01 & 0.0805 & 0.511501 & -22.17 & 0.49 & 1754 & -0.60 \\
$97-139$ & granite & 1440 & 35.82 & 5.23 & 0.08834 & 0.511602 & -20.21 & -0.21 & 1743 & -0.56 \\
$97-145$ & granodiorite & 1562 & 38.91 & 5.89 & 0.09165 & 0.511605 & -20.16 & 0.89 & 1786 & -0.53 \\
$97-147$ & granod. gneiss & 1586 & 40.40 & 6.72 & 0.10064 & 0.511639 & -19.48 & 0.06 & 1882 & -0.49 \\
$97-150$ & tonalite & 1549 & 28.41 & 5.53 & 0.11774 & 0.511884 & -14.70 & 0.98 & 1829 & -0.40 \\
\hline
\end{tabular}

the Figure 4B (alumina index; Maniar and Piccoli 1989), the studied samples vary from peraluminous (granitic and granodioritic rocks) to metaluminous (tonalitic rocks).

The hypothesis of fractionation is confirmed by $\mathrm{Rb} / \mathrm{Sr}$ versus $\mathrm{Sr}$ diagram (Figure 5) which analytical data plot indicates an increasing $\mathrm{Rb} / \mathrm{Sr}$ ratio and decreasing Sr content. The post-tectonic plutonic rocks (U-Pb ages from 1.48 to $1.39 \mathrm{Ga}$ ) show $\mathrm{SiO}_{2}$ variation from $73 \%$ to $70 \%$, with low $\mathrm{Ca}_{2} \mathrm{O}$ content (from $3 \%$ to $1.37 \%$ ) and high $\mathrm{Na}_{2} \mathrm{O}$ and $\mathrm{K}_{2} \mathrm{O}$ val- ues $(4.3 \%$ to $3.7 \%$ and $4.6 \%$ to $2.6 \%$ ), indicating that this group of rocks present highly fractionated signature.

The REE patterns indicate a higher fractionation between LREE and HREE for the felsic rocks (97-138, 97-132, 97-129 and 97-139), compared with the intermediate (97-147, 97-145 and 97-136) and primitive rocks (97-134 and 97-150). The REE distribution between the rocks is also characterized by slightly positive Eu anomaly in the most primitive rocks, a slightly negative Eu anomaly in the inter- 

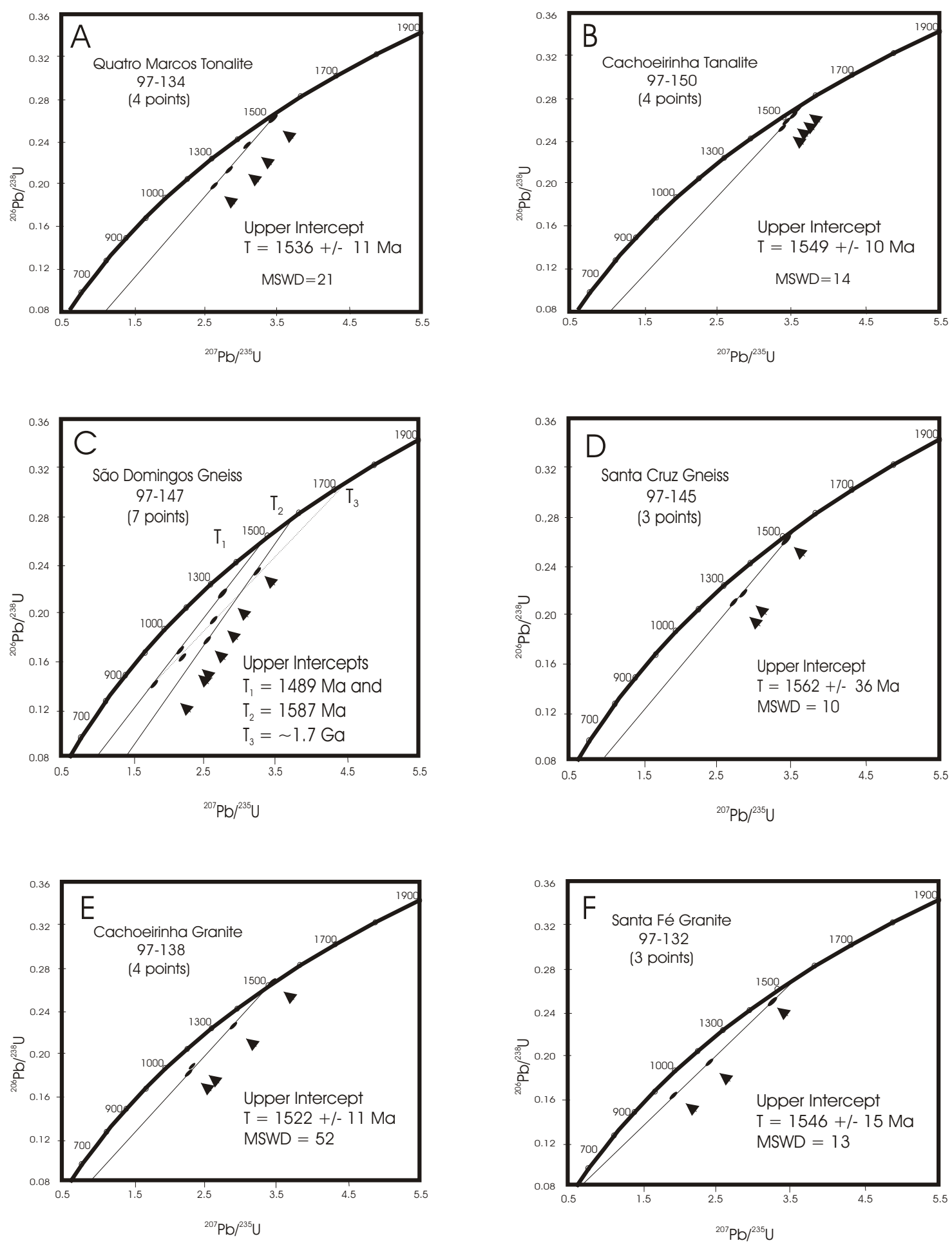

Fig. 2 - Concordia diagrams for the tonalitic (samples 97-134 and 97-150), granodioritic (samples 97-149 and 97-145) and granitic rocks (samples 97-138 and 97-132) of the Cachoeirinha suite. 

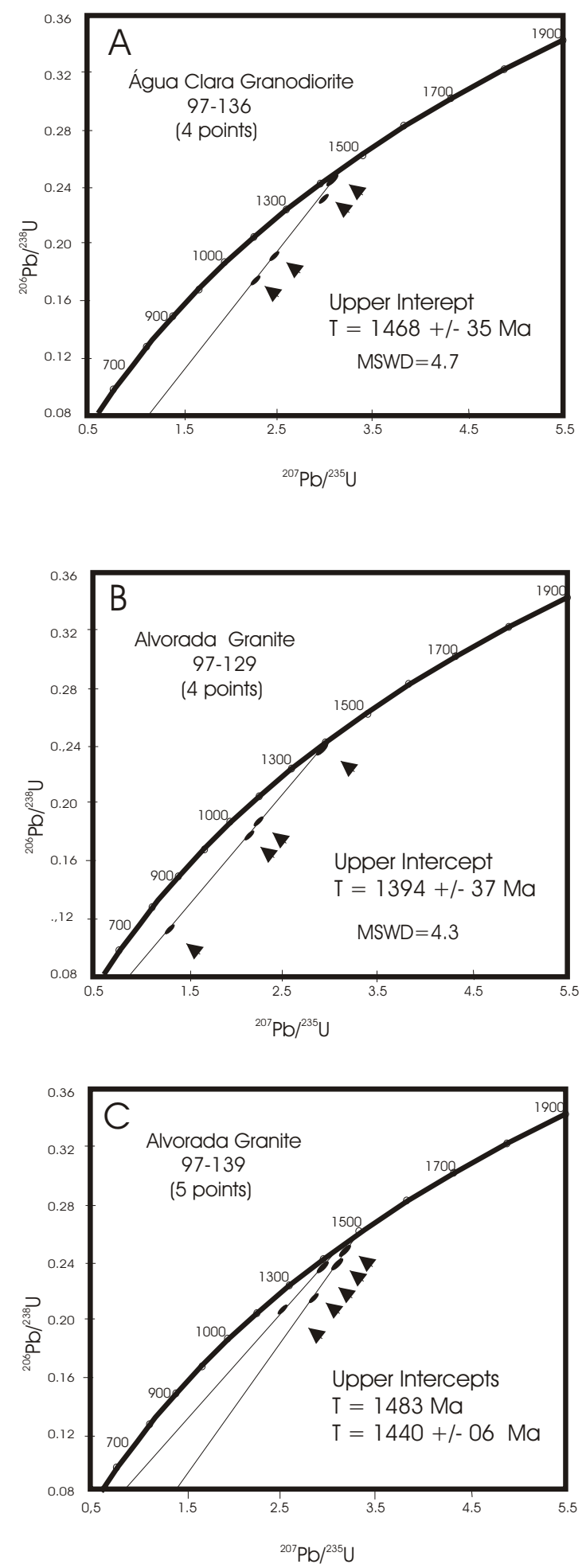

Fig. 3 - Concordia diagrams for the post-tectonic granodioritic (sample 96-136) and granitic (samples 97-129 and 97-139) intrusive rocks of the Cachoeirinha suite. 

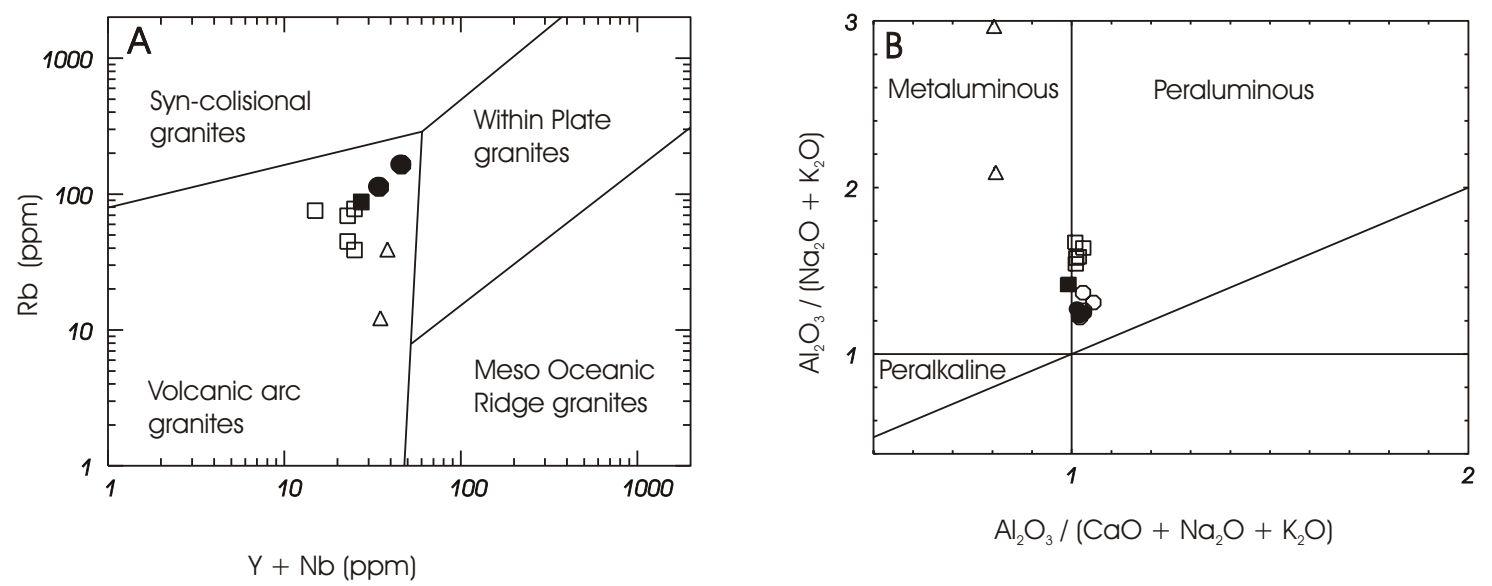

Fig. 4 - (A) Tectonic discrimination diagram (Pearce et al. 1984) and (B) Shandy Index (Maniar and Piccoli 1989) for Cachoeirinha suite rocks. Open symbols $=$ post-orogenic rocks; and filled symbols $=$ orogenic rocks.

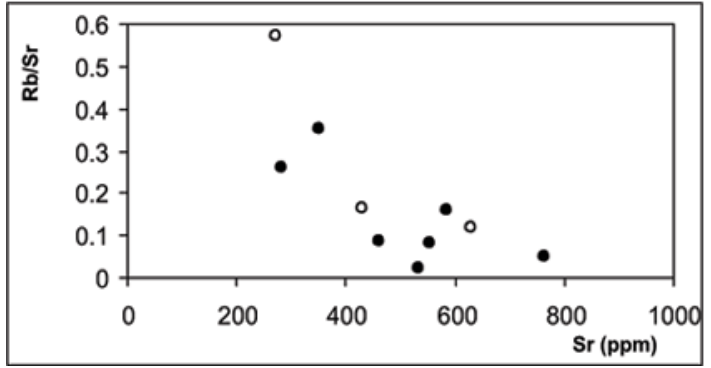

Fig. $5-\mathrm{Rb} / \mathrm{Sr}$ versus $\mathrm{Sr}$ diagram. The rapidly increasing $\mathrm{Rb} / \mathrm{Sr}$ ratio with decreasing $\mathrm{Sr}$ content can be explained by removal of plagioclase. See text for discussion. Open symbols = postorogenic rocks; and filled symbols $=$ orogenic rocks.

mediate ones, and moderate Eu negative anomaly in the felsic rocks (Figure 6).

The post-orogenic granites show two distinctive REE patterns (Figure 6). The Agua Clara granodiorite (sample 97-136) presents REE distribution similar to the primitive rocks of the Cachoeirinha suite rocks, and the other two samples (97-129 and 97139) show REE patterns similar to the felsic rocks of the Cachoeirinha suite rocks.

\section{DISCUSSION}

Geochronological, isotopic and chemical data provide informations about the nature and tectonic set-

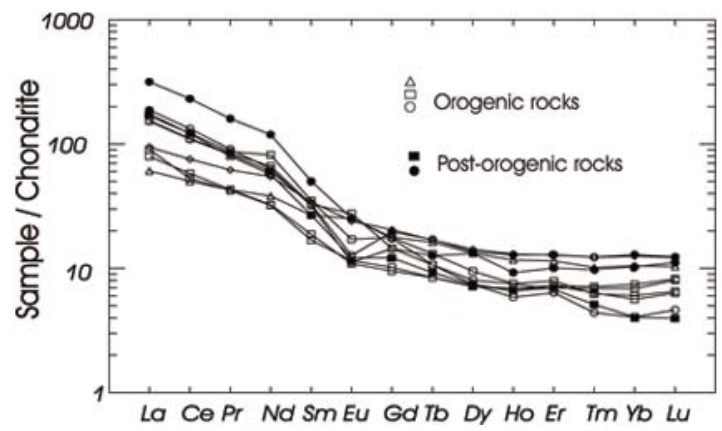

Fig. 6 - REE patterns for samples of Cachoeirinha suite rocks. Chondrite normalized according to Taylor and McLennan (1985). Triangle: tonalites; Square: granodiorites; and Circle: granites.

ting of the Cachoeirinha suite, which have important bearing for the understanding of the crustal evolution of the SW Amazonian craton at Mesoproterozoic time. U-Pb zircon ages indicate that the $\mathrm{Ca}$ choeirinha granitoids comprise a voluminous rock suite formed during a short period of time (1590 Ma to $1520 \mathrm{Ma}$ ), implying that a regional tectonic magmatic event took place succeeding the generation of the Alto Jauru volcanic-plutonic accretionary complex (Geraldes et al. 2001). The $\mathrm{T}_{\mathrm{DM}}$ between $1.88 \mathrm{Ga}$ and $1.75 \mathrm{Ga}$ and the $\varepsilon_{\mathrm{Nd}(\mathrm{t})}$ values varying from slightly negative $(-0.8)$ to positive $(+1.0)$ for the investigated Cachoeirinha rocks together with their calc-alkaline affinity is consistent with an arc- 
TABLE V

U-Pb isotopic results from Cachoeirinha suite rocks.

\begin{tabular}{|c|c|c|c|c|c|c|c|c|c|}
\hline Sample & $97-129$ & $97-130$ & $97-132$ & $97-136$ & $97-138$ & $97-139$ & $97-145$ & $97-149$ & $97-150$ \\
\hline $\mathrm{SiO}_{2}$ & 73.38 & 44.07 & 73.26 & 70.74 & 73.37 & 70.77 & 68.26 & 71.12 & 55.38 \\
\hline $\mathrm{Al}_{2} \mathrm{O}_{3}$ & 14.05 & 16.63 & 14.21 & 15.55 & 14.04 & 15.34 & 15.73 & 14.77 & 16.21 \\
\hline $\mathrm{Fe}_{2} \mathrm{O}_{3}$ & 1.94 & 16.98 & 2.06 & 2.61 & 2.05 & 2.93 & 3.94 & 3.84 & 9.06 \\
\hline $\mathrm{MnO}$ & 0.05 & 0.19 & 0.05 & 0.04 & 0.03 & 0.05 & 0.05 & 0.08 & 0.14 \\
\hline $\mathrm{MgO}$ & 0.32 & 7.17 & 0.47 & 0.78 & 0.40 & 0.83 & 1.02 & 0.91 & 4.76 \\
\hline $\mathrm{CaO}$ & 1.37 & 8.29 & 1.89 & 2.92 & 1.30 & 2.99 & 2.62 & 3.19 & 7.04 \\
\hline $\mathrm{Na}_{2} \mathrm{O}$ & 3.70 & 2.86 & 3.62 & 4.34 & 3.48 & 4.19 & 4.61 & 4.21 & 3.39 \\
\hline $\mathrm{K}_{2} \mathrm{O}$ & 4.66 & 0.83 & 4.09 & 2.72 & 5.14 & 2.62 & 2.79 & 1.77 & 1.91 \\
\hline $\mathrm{TiO}_{2}$ & 0.21 & 2.26 & 0.23 & 0.33 & 0.22 & 0.33 & 0.52 & 0.28 & 1.10 \\
\hline $\mathrm{P}_{2} \mathrm{O}_{5}$ & 0.08 & 0.30 & 0.08 & 0.11 & 0.09 & 0.11 & 0.21 & 0.12 & 0.48 \\
\hline LOI & 0.23 & 0.31 & 0.21 & 0.34 & 0.24 & 0.25 & 0.21 & 0.32 & 0.42 \\
\hline total & 99.76 & 99.58 & 99.96 & 100.14 & 100.12 & 100.16 & 99.75 & 100.29 & 99.47 \\
\hline $\mathrm{Be}$ & 3 & 2 & 2 & 2 & 1 & 2 & 1 & 2 & 2 \\
\hline $\mathrm{V}$ & 17 & 358 & 9 & 32 & 8 & 26 & 52 & 33 & 169 \\
\hline $\mathrm{Cr}$ & 0 & 59 & 0 & 0 & 0 & 0 & 0 & 0 & 89 \\
\hline Co & 1.9 & 76 & 2.6 & 5.4 & 2.5 & 5 & 6.8 & 6.6 & 27 \\
\hline $\mathrm{Ni}$ & 0 & 147 & 24 & 0 & 0 & 0 & 0 & 0 & 44 \\
\hline $\mathrm{Cu}$ & 0 & 42 & 0 & 0 & 0 & 0 & 0 & 0 & 42 \\
\hline $\mathrm{Zn}$ & 40 & 138 & 46 & 65 & 37 & 68 & 60 & 51 & 102 \\
\hline $\mathrm{Ga}$ & 18 & 24 & 17 & 19 & 14 & 19 & 16 & 14 & 20 \\
\hline $\mathrm{Ge}$ & 1.3 & 1.5 & 1.2 & 1 & 1.1 & 1 & 0.8 & 1.3 & 1.3 \\
\hline As & 0 & 0 & 0 & 0 & 0 & 0 & 0 & 0 & 0 \\
\hline $\mathrm{Rb}$ & 154 & 13 & 123 & 76 & 74 & 71 & 46 & 39 & 40 \\
\hline $\mathrm{Sr}$ & 270 & 532 & 350 & 628 & 282 & 429 & 552 & 459 & 763 \\
\hline $\mathrm{Y}$ & 30 & 26 & 23 & 22 & 14 & 16 & 19 & 17 & 30 \\
\hline $\mathrm{Zr}$ & 175 & 137 & 163 & 122 & 187 & 172 & 236 & 90 & 133 \\
\hline $\mathrm{Nb}$ & 17 & 7.4 & 9.2 & 4.3 & 2.6 & 7.5 & 3.7 & 6 & 6.7 \\
\hline Mo & 0.4 & 0.8 & 0.3 & 0.7 & 0.4 & 0.7 & 0.6 & 0.7 & 0.7 \\
\hline $\mathrm{Ag}$ & 0 & 0 & 0 & 0 & 0 & 0 & 0 & 0 & 0 \\
\hline In & 0 & 0 & 0 & 0 & 0 & 0 & 0 & 0 & 0 \\
\hline $\mathrm{Sn}$ & 1.5 & 1.6 & 1.5 & 1.9 & 0.7 & 1.5 & 0.7 & 0.6 & 1.2 \\
\hline $\mathrm{Sb}$ & 0.13 & 0.09 & 0.18 & 0.07 & 0.21 & 0.1 & 0.09 & 0.07 & 0.09 \\
\hline $\mathrm{Cs}$ & 1 & 0.4 & 0.9 & 2.3 & 0.4 & 0.6 & 0.4 & 0.4 & 0.5 \\
\hline $\mathrm{Ba}$ & 1160 & 432 & 1505 & 999 & 2376 & 1210 & 2134 & 840 & 1002 \\
\hline $\mathrm{Hf}$ & 5.3 & 3.5 & 4.8 & 3.2 & 4.7 & 4.6 & 5.8 & 2.6 & 3.7 \\
\hline $\mathrm{Ta}$ & 1.18 & 0.39 & 0.61 & 0.29 & 0.22 & 0.52 & 0.17 & 0.52 & 0.22 \\
\hline W & 0.6 & 1 & 0 & 0.4 & 3 & 0 & 0.2 & 0.3 & 0.5 \\
\hline $\mathrm{Tl}$ & 0.92 & -0.05 & 0.95 & 0.86 & 0.4 & 0.71 & 0.26 & 0.19 & 0.32 \\
\hline $\mathrm{Pb}$ & 35 & 7 & 38 & 14 & 32 & 28 & 19 & 11 & 12 \\
\hline
\end{tabular}


TABLE V (continuation)

\begin{tabular}{l|c|c|c|c|c|c|c|c|c}
\hline Sample & $97-129$ & $97-130$ & $97-132$ & $97-136$ & $97-138$ & $97-139$ & $97-145$ & $97-149$ & $97-150$ \\
\hline $\mathrm{Bi}$ & 05 & 0 & 0 & 0 & 0 & 0 & 0 & 0 & 0.08 \\
$\mathrm{Th}$ & 24.1 & 1.52 & 23.4 & 3.67 & 16.3 & 19.6 & 5.75 & 5.14 & 3.78 \\
$\mathrm{U}$ & 8.64 & 0.32 & 3.33 & 1.13 & 2.07 & 3.06 & 1.11 & 1.11 & 0.75 \\
$\mathrm{La}$ & 50.3 & 20 & 61.9 & 29.7 & 104 & 57.8 & 50.9 & 26.2 & 31 \\
$\mathrm{Ce}$ & 94.3 & 43.4 & 115 & 45.7 & 200 & 105 & 95.6 & 50.1 & 65.4 \\
$\mathrm{Pr}$ & 10.77 & 5.566 & 11.83 & 5.511 & 20.79 & 10.39 & 10.93 & 5.595 & 8.027 \\
$\mathrm{Nd}$ & 37.9 & 24.1 & 39.7 & 20.2 & 75 & 36.1 & 41.7 & 20.5 & 34.5 \\
$\mathrm{Sm}$ & 6.86 & 5.42 & 6.5 & 3.43 & 10.2 & 5.46 & 6.67 & 3.8 & 6.95 \\
$\mathrm{Eu}$ & 0.977 & 1.941 & 0.862 & 0.889 & 1.948 & 0.934 & 2.12 & 0.837 & 1.845 \\
$\mathrm{Gd}$ & 5.42 & 4.9 & 3.97 & 2.8 & 4.8 & 3.35 & 4.01 & 2.63 & 5.65 \\
$\mathrm{~Tb}$ & 0.85 & 0.81 & 0.63 & 0.42 & 0.53 & 0.46 & 0.53 & 0.42 & 0.84 \\
$\mathrm{Dy}$ & 4.67 & 4.53 & 3.52 & 2.45 & 2.54 & 2.55 & 2.75 & 2.49 & 4.87 \\
$\mathrm{Ho}$ & 0.99 & 0.9 & 0.71 & 0.53 & 0.45 & 0.51 & 0.58 & 0.51 & 1 \\
$\mathrm{Er}$ & 2.89 & 2.58 & 2.26 & 1.64 & 1.43 & 1.57 & 1.78 & 1.64 & 2.88 \\
$\mathrm{Tm}$ & 0.433 & 0.358 & 0.344 & 0.224 & 0.155 & 0.242 & 0.219 & 0.25 & 0.433 \\
$\mathrm{Yb}$ & 2.82 & 2.3 & 2.26 & 1.24 & 0.89 & 1.51 & 1.33 & 1.63 & 2.75 \\
$\mathrm{Lu}$ & 0.418 & 0.351 & 0.378 & 0.215 & 0.157 & 0.272 & 0.221 & 0.278 & 0.415 \\
\hline
\end{tabular}

related setting for this unit. Moreover, the $\mathrm{Nd}$ evidence (Figure 7) suggests crustal contribution in the genesis of the Cachoeirinha suite. This is corroborated by the U-Pb results of sample 97-149, with several inherited zircon populations, probably due to contribution of Alto Jauru crust in the source for this rock. If this interpretation is correct, the sample 97-149 grains of zircon may have retained isotopic signature of ancient rocks, and the magmatic event responsible for the Cachoeirinha suite formation kept part of these zircon within the analyzed sample. The alignment of four U-Pb analytical results of this sample yielded an age of about $1.7 \mathrm{Ga}$ (Figure 2C), suggesting that the older protholith may be the Alto Jauru country rocks.

Post-orogenic magmatism represented by samples 97-129, 97-136 and 97-139 shows crystallization U-Pb zircon ages (Figures 3A, 3B and 3C) varying from $1485 \mathrm{Ma}$ to $1389 \mathrm{Ma}$, with correspondent Sm-Nd results $\left(\varepsilon_{\mathrm{Nd}(t)}\right.$ values between -1.3 and +1.7$)$ indicating a probable participation of the Alto Jauru rocks in their sources.

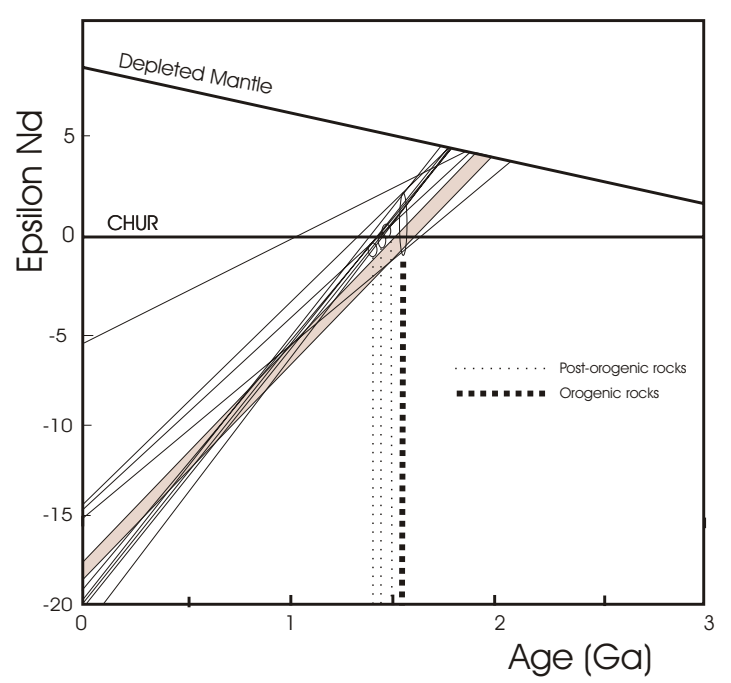

Fig. 7-Nd isotopic evolution of Cachoeirinha rocks. The Nd isotopic evolution curves of Alto Jauru rocks (basement) are plotted in gray (according to Geraldes et al. 2001). 


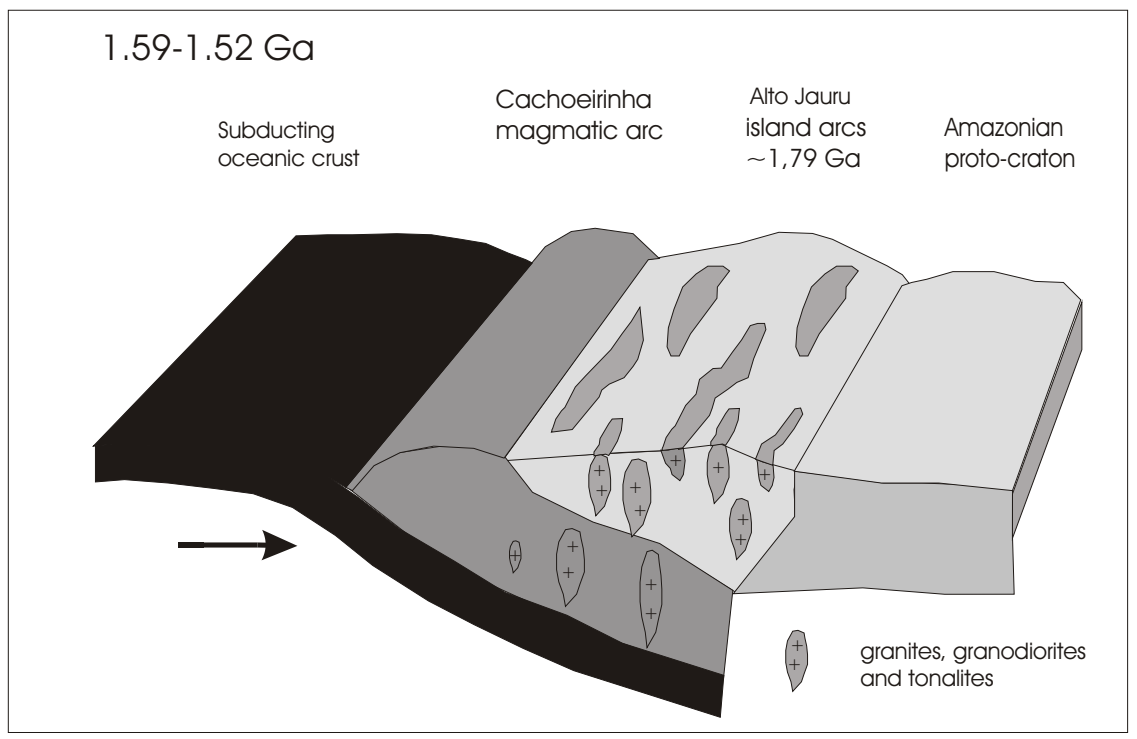

Fig. 8 - Crustal evolution model for the Cachoeirinha arc at about 1.59-1.52 Ga.

The Cachoeirinha suite rocks $\mathrm{SiO}_{2}$ contents range from about $68 \%$ to $73 \%$, contains primitive (high-Ca), and highly evolved (high-K, Rb) phases. The post-tectonic rocks (samples 97-136, 97-139 and 97-129) are the highest evolved rocks and they show $\mathrm{SiO}_{2}$ contents from $70 \%$ to $73 \%$. The $\mathrm{Ca}$ choeirinha granitoids show I-type affinities for the primitive and intermediate rocks and A-type affinities for the pos-tectonic more evolved rocks (Figure 4A) and chemical results plot near the peraluminousmetaluminous boundary on the $\mathrm{A} / \mathrm{CNK}$ versus ZNCY diagram (Figure 4B). REE data for the Cachoeirinha suite have steep LREE patterns and relatively flat HREE patterns with moderately negative to absent Eu anomalies. Both major and trace elements in the analyzed samples show distributions characteristic of fractional crystallization. As example, the increasing $\mathrm{Rb} / \mathrm{Sr}$ ratio with decreasing Sr content (Figure 5) can be explained by removal of plagioclase, K-feldspar, and biotite in the approximate proportions that they occur in the rocks.

The Cachoeirinha suite presents isotopic and chemical signatures that define their juvenile character. Consequently we propose that the rock association here reported was formed in a magmatic arc setting, (e.g., Cachoeirinha magmatic arc) close to an older continental crust (as speculated in Figure 8) during an orogenic event that occurred in the SW (actual) margin of the Amazonian craton which magmatism source had an important contribution of the older crust comprised by the 1790-1740 Ma Alto Jauru volcano-plutonic rocks.

Cachoeirinha rocks may be correlated to rocks formed in coeval magmatic arcs described in Baltica, where continent-continent collision occurred at ca. $1.58 \mathrm{Ga}$ and eastward subduction was renewed (Åhäll and Gower 1997). Evidence is given by calc-alkaline ca. $1.55 \mathrm{Ga}$ mafic-ultramafic tonalitic intrusions (northern Telemark) and ca. 1.53$1.50 \mathrm{Ga}$ rapakivi magmatism in central Sweden. These events are coeval and consistent with the onset of the Cachoeirinha orogen in SW Mato Grosso, and in Finland, by the Aland Riga Group (1.58-1.54 Ga) and ca. 1.56-1.54 Ga Salmi Group rapakivi plutons (Rämö and Haapala 1995). Similarly, the 1.59-1.52 Ga age pattern of the Cachoeirinha suite is partially coeval with the 1.61-1.53 Ga Serra da Providência suite, described as a bimodal (rapakivi granites and gabbros) intrusive suite, according to Bettencourt et al. (1999). 
Åhäll et al. (2000) reported more detailed U$\mathrm{Pb}$ studies in the Baltica Mesoproterozoic granites and defined two accretionary events: The first event coined Stage 2 formed crust from 1.62 to $1.58 \mathrm{Ga}$ and Stage 3 formed crust from 1.56 to $1.55 \mathrm{Ga}$. These authors concluded that the rapakivi suites to the east are inboard manifestations of coeval accretionary process in the west. The data reported by Åhäll et al. (2000) added to data here reported suggest that Amazonia and Baltica had coeval juvenil accretionary events, indicating that both continental masses could be connected as a laterally continuous continental margin at 1.6 to $1.5 \mathrm{Ga}$.

\section{CONCLUSIONS}

$\mathrm{U}-\mathrm{Pb}$ and $\mathrm{Sm}-\mathrm{Nd}$ major, trace and REE data provide time and tectonic setting constraints for the rocks here studied and allow to suggest tectonic implications on the Mesoproterozoic crustal evolution of the SW Amazonian craton, as the following:

1) Tonalites, granodiorites and granites ascribed to the Cachoeirinha suite represent juvenile magmatic activity that occurred between 1590 and $1520 \mathrm{Ma}$. Thereby slightly younger than the Santa Helena arc (1.45-1.42 Ga), the improved chronological resolution obtained using $\mathrm{U}-\mathrm{Pb}$ and $\mathrm{Sm}-\mathrm{Nd}$ methods, added to integrated geochemical data, allows to conclude that the rock association are components of a ca. $1550 \mathrm{Ma}$ NW-trending plutonic arc $(\mathrm{Ca}-$ choeirinha arc).

2) The $T_{D M}$ model ages for rocks of the Cachoeirinha suite, mostly between 1.83-1.75 Ga, suggest some participation of an older crust, in concordance with the development of such arc complex along the western margin (actual) of a Mesoproterozoic continental crust composed by the 1.79-1.74 Ga Alto Jauru volcanoplutonic rocks. However $\mathrm{T}_{\mathrm{DM}}$ of $2.05 \mathrm{Ga}$ observed in sample 97-149 may indicate a component with age older than Alto Jauru crust.

3) The age pattern of 1.59-1.52 Ga rocks intruded into or adjacent to an older continental crust is similar to geologic framework described along the eastern and southern margins of Baltica prior to $1500 \mathrm{Ma}$, and would be compatible with tectonic models which propose proximity between Laurentia, Baltica and Amazonia at about $1.6 \mathrm{Ga}$ to $1.5 \mathrm{Ga}$.

\section{ACKNOWLEDGMENTS}

This paper was improved by discussion with J.S. Bettencourt (Universidade de São Paulo-SP Brazil) and R. Dall'Agnol (Universidade Federal do Pará-PA - Brazil). This work was sponsored by Fundação de Amparo à Pesquisa do Estado de São Paulo (FAPESP) Grant 96-04819-7 to M.C. Geraldes and Grant 96-9022-0 to W. Teixeira. This paper is a contribution to IGCP-426: Granite Systems and Proterozoic Lithospheric Processes.

\section{RESUMO}

Estudos isotópicos e químicos foram realizados em rochas da Suíte Cachoeirinha, SW do craton Amazônico. Idades $\mathrm{U}-\mathrm{Pb}$ e $\mathrm{Sm}-\mathrm{Nd}$ em rochas granitóides desta suíte forneceram idades de cristalização entre 1587-1522 Ma e idades modelo $\mathrm{T}_{\mathrm{DM}}$ entre $1,88-1,75 \mathrm{Ga}\left(\varepsilon_{\mathrm{Nd}}\right.$ entre $\left.-0,8 \mathrm{a}+1,0\right)$. Em adição, rochas intrusivas apresentam idades U-Pb entre 1485-1389 Ma e apresentam $\mathrm{T}_{\mathrm{DM}}$ entre 1,77-1,74 Ga e valores de $\varepsilon_{\mathrm{Nd}}$ entre $-1,3$ e +1.7 . Variações químicas da suíte Cachoeirinha sugerem a existência de processo de cristalização fracionada em ambiente de arco magmático. Os resultados aqui reportados permitem as seguintes conclusões: (1) no período de tempo entre 1590-1520 Ma ocorreu um importante magmatismo no SW do craton Amazônico; (2) as assinaturas isotópicas de Nd e dados químicos suportam a hipótese de que as rochas plutônicas foram geradas em ambiente de arco magmático relacionado a uma subducção sob a margem continental representada pelas rochas do Alto Jauru com idades de 1,791,74 Ga; (3) Idades entre 1590-1520 Ma de rochas intrusivas adjacente a uma crosta mais antiga sugerem um contexto geológico semelhante ao longo da margem sul da Laurentia-Báltica no período de tempo aqui estudado.

Palavras-chave: Craton Amazônico, geocronologia U$\mathrm{Pb}$ e Sm-Nd, Mesoproterozóico, magmatismo de arco. 


\section{REFERENCES}

ÅHÄLl KI AND Gower CF. 1997. The Gotian and Labradorian orogens: variations in accretionary tectonism along a late Paleoproterozoivc LaurentiaBaltica margin. Geol Fören Stockolm Förh 119: 181-191.

ÅHäll KI, Connelly JN AND BRewer TS. 2000. Episodic rapakivi magmatism due to distal orogenesis?: correlation of 1.69-1.50 Ga orogenic and inboard, " anorogenic'" events in the Baltic Shield. Geology 28: 823-826.

Bettencourt JS, Tosdal RM, Leite JR WR AND Payolla BL. 1999. Mesoproterozoic rapakivi granites of Rondônia Tin Province, southwestern border of Amazonian craton, Brazil-I: Reconnaissance U-Pb geochronology and regional implications. Precamb Res 95: 41-67.

Carneiro MA, Ulbrich HHGJ and Kawashita K. 1992. Proterozoic crustal evolution at the southern margin of the Amazonian craton in the State of Mato Grosso, Brazil: evidence from $\mathrm{Rb}-\mathrm{Sr}$ and $\mathrm{K}-\mathrm{Ar}$ data. Precamb Res 59: 263-282.

D’Agrella MS, Pacca IGI, Siqueira R, Elming SA, Teixeira W, Bettencourt JS and Geraldes MC. 2000. Amazonian Proterozoic poles: Implications to Rodinia paleogeography. In: From basins to mountains: Rodinia at the turn of the century. K.N. Sircombe and Z.X. Li. Geological Society of Australia Abstracts 65: 27-30.

DALzIEL IWD. 1992. On the organization of American plates in the Neoproterozoic and the breakup of Laurentia. GSA Today 2: 237-241.

DAlziel IWD. 1997. Antarctica: A tale of two supercontinent? Earth Planet Sci Let 20: 501-526.

DePaolo DJ. 1988. Neodymium Isotope Geochemistry. Springer-Verlag, $187 \mathrm{p}$.

Geraldes MC, Figueiredo BR, Tassinari CCG AND Ebert HD. 1997. Middle Proterozoic vein-hosted gold deposits in the Pontes e Lacerda region, southwestern Amazonian craton, Brazil. Int Geol Rev 39: 438-448.

Geraldes MC, Van Schmus WR, Condie KC, Bell S, Teixeira W and Babinski M. 2001. Proterozoic Geologic Evolution of the SW Part of the Amazonian Craton in Mato Grosso State, Brazil. Precamb Res 111: 91-128.
Geraldes MC, Tassinari CCG, Teixeira W and Van Schmus WR. 2002. Geocronologia U-Pb convencional e SHRIMP e Sm-Nd de rochas granitóides na Serra Santa Bárbara (SW do Estado do Mato Grosso): Uma possível extensão do orógeno San Ignácio da Bolívia (?). Contribuições à Geologia da Amazônia 3: $143-151$.

Gower CF AND Tucker RD. 1994. Distribution of pre1400 Ma crust in the Greenville Province: Implications for rifting in Laurentia-Baltica during Geon 14. Geology 22: 827-830.

HofFMAN PF. 1991. Did the breakout of Laurentia turn Gondwanaland inside-out? Science 252: 14091412.

Janasi VA, ANdrade S and Ulbrich HHGJ. 1996. A correção do drift instrumental do ICP-AS com espectrometria sequencial e a análise de elementos maiores, menores, e traços em rochas. Boletim do I.G. Série Científica 26: 45-58.

KROGH TE. 1973. A low contamination method for hydrothermal decomposition of zircon and extraction of $\mathrm{U}$ and $\mathrm{Pb}$ for isotopic age determinations. Geochimica et Cosmochimica Acta 37: 485-494.

KROGH TE. 1982. Improved accuracy of U-Pb zircon ages by the creation of more concordant systems using an air abrasion technique. Geochimica et Cosmochimica Acta 46: 637-649.

Litherland M, Annels RN, Appleton JD, Berrange JP, Bloomfield K, Burton CCJ, Darbyshire DPF, Fletcher CJN, Hawkins MP, Klink BA et al. 1986. The Geology and Mineral Resources of the Bolivian Precambrian Shield. British geological Survey. Overseas Memoir 9. London, Her Majesty's Stationery Office, $140 \mathrm{p}$.

Litherland M, Annels RN, Darbyshire DPF, Fletcher CJN, Hawkins MP, Klink BA, Mitchel WI, Occonnors EA, Pitfield PEJ, Power G AND WerB BC. 1989. The Proterozoic eastern Bolivia and its relationship to the Andean mobile belt. Precamb Res 43: 157-174.

LudWIG KR. 1998. Using Isoplot/Ex, version 1.00b, a geochronological toolkit for Microsoft Excel. Berkeley Geochronology Center Spec Pub, No. 1, 43 p.

Maniar PD and Piccoli PM. 1989. Tectonic discrimination of granitoids. Geol Soc Amer Bull 101: 535-543. 
Matos JB, Schorscher JHD, Geraldes MC, Souza MZA AND RuIz AS. 2004. Petrografia, geoquímica e geocronologia das rochas do orógeno Rio Alegre, Mato Grosso: um registro de crosta oceânica Mesoproterozóica no SW do craton Amazônico. Geologia USP (Revista do Instituto de Geociências-USP). Série Científica 4: 75-90.

Moores EM. 1991. Southeast U.S.-east Antactica (SWEAT) connection: A hypothesis: Geology 19: 425-428.

Neder RD, Leite JAD, Figueiredo BR and MCNAUghton NJ. 2002. 1.76 Ga volcano-plutonism in the southwestern Amazonian craton, AripuanãMT, Brazil: tectono-stratigraphic implications from SHRIMP U-Pb zircon data and rock geochemistry, Precamb Res 119: 171-187.

PARRISH RR. 1987. An improved micro-capsule for zircon dissolution in $\mathrm{U}-\mathrm{Pb}$ geochronology. Isotope Geosciences 66: 99-102.

Patchett PJ ANd Ruiz J. 1987. Nd isotopic ages of crust formation and metamorphism in the Precambrian of eastern and southern Mexico. Contrib Mineral Petrol 96: 523-528.

Pearce JA, Harris NBW and Tindle AG. 1984. Trace element discrimination diagrams for the tectonic interpretation of granitic rocks. J Petrol 25: 956-983.

Pinho FEC, Fyfe WS and Pinho MASB. 1997. Early Proterozoic evolution of the Alto Jauru greenstone belt, southern Amazonian craton, Brazil. Int Geol Rev 39: 220-229.

RÄмÖ OT AND HaAPALA I. 1995. One hundred years of rapakivi granite. Mineral Petrol 52: 129-185.

ROGERS JW. 1996. A history of continents in the past three billions years. J Geol 104: 91-107.

SAdOwSKy GR ANd BetTenCourt JS. 1996. Mesoproterozoic tectonic correlations between east Laurentia and the western border of the Amazon Craton. Precamb Res 76: 213-227.

Santos JOS, Hartman LA, Gaudete HE, Groves DI, McNaughton NJ and Fletcher IR. 2000. A new undertanding of the provinces of the Amazonian craton based on integration of field mapping and U$\mathrm{Pb}$ and $\mathrm{Sm}-\mathrm{Nd}$ geochronology. Gondwana Res 3: 489-506.
Sato K And Tassinari CCG. 1997. Principais eventos de acreção continental no Cráton Amazônico baseados em idade modelo Sm-Nd, calculada em evoluções de estágio único e estágio duplo. In: Costa ML AND ANGÉliCa RS, eds. Contribuições à Geologia da Amazônia, Belém, Sociedade Brasileira de Geociências, p. 91-142.

Stacey JS AND Kramers JD. 1975. Approximation of terrestrial lead isotope evolution by a two stage model. Earth Planet Sci Let 26: 207-221.

Steiger RH AND JÄGER E. 1977. Subcommission on Geochronology-convention and use of decay constants in geochronology and cosmochronology. Earth Planet Sci Let 36: 359-362.

Tassinari CCG and Macambira MJB. 1999. Geochronological Provinces of the Amazonian Craton. Episodes 22: 174-182.

Tassinari CCG, Cordani UG, Nutman A, Van Schmus WR, Bettencourt JS and Taylor PN. 1997. Geochronological systematics on basement rocks from the Rio Negro-Juruena Province (Amazon Craton), and tectonic implications. Int Geol Rev 35: 263-277.

Tassinari CCG, Bettencourt JS, Geraldes MC, Macambira MJB and Lafon JM. 2000. The Amazon craton. In Cordani U, Milani EJ, Thomaz Filho A and Campos DA, eds. Tectonic evolution of South America, 31 International Geological Congress, Rio de Janeiro-Brazil, p. 41-95.

TAYlor SR And McLennan SM. 1985. The Continental Crust: Its composition and evolution. Blackwell, Oxford, $312 \mathrm{p}$.

Teixeira W, Tassinari CCG, Cordani UG and KaWAShita K. 1989. A review of the geochronology of the Amazonian craton: tectonic implications. Precamb Res 42: 213-227.

Tohver E, Van der Pluijm B, Scandolara J and Rizzotto G. 2002. Paleogeography of the Amazon craton at $1.2 \mathrm{Ga}$ : early Grenvillian collision with the Llano segment of Laurentia. Earth Planet Sci Let 199: 185-200.

UNRUG R. 1996. The assembly of Gondwanaland. Episodes 19: 11-20. 